University of Wollongong

Research Online

Australian Institute for Innovative Materials -

Papers

Australian Institute for Innovative Materials

$1-1-2016$

Brazing techniques for the fabrication of biocompatible carbon-based electronic devices

Nicholas V. Apollo

University of Melbourne

Desmond Lau

Royal Melbourne Institute of Technology

Arman Ahnood

University of Melbourne

Alastair Stacey

University of Melbourne

Kumaravelu Ganesan

University of Melbourne

See next page for additional authors

Follow this and additional works at: https://ro.uow.edu.au/aiimpapers

Part of the Engineering Commons, and the Physical Sciences and Mathematics Commons

Research Online is the open access institutional repository for the University of Wollongong. For further information contact the UOW Library: research-pubs@uow.edu.au 


\title{
Brazing techniques for the fabrication of biocompatible carbon-based electronic devices
}

\author{
Abstract \\ Prototype electronic devices have been critical to the discovery and demonstration of the unique \\ properties of new materials, including composites based on carbon nanotubes (CNT) and graphene. \\ However, these devices are not typically constructed with durability or biocompatibility in mind, relying on \\ conductive polymeric adhesives, mechanical clamps or crimps, or solders for electrical connections. In \\ this paper, two key metallization techniques are presented that employ commercially-available brazing \\ alloys to fabricate electronic devices based on diamond and carbonaceous wires. Investigation of the \\ carbon - alloy interfacial interactions was utilized to guide device fabrication. The interplay of both \\ chemical ("adhesive") and mechanical ("cohesive") forces at the interface of different forms of carbon \\ was exploited to fabricate either freestanding or substrate-fixed carbonaceous electronic devices. \\ Elemental analysis in conjunction with scanning electron microscopy of the carbon - alloy interface \\ revealed the chemical nature of the Ag alloy bond and the mechanical nature of the Au alloy bond. \\ Electrical characterization revealed the non-rectifying nature of the carbon - Au alloy interconnects. \\ Finally, electronic devices were fabricated, including a Au circuit structure embedded in a polycrystalline \\ diamond substrate.

\section{Disciplines} \\ Engineering | Physical Sciences and Mathematics

\section{Publication Details} \\ Apollo, N. V., Lau, D., Ahnood, A., Stacey, A., Ganesan, K., Lichter, S. G., Fox, K., Foroughi, J., Meffin, H., \\ Wallace, G. G., Baughman, R. H., Prawer, S. \& Garrett, D. J. (2016). Brazing techniques for the fabrication of \\ biocompatible carbon-based electronic devices. Carbon, 107 180-189.

\section{Authors} \\ Nicholas V. Apollo, Desmond Lau, Arman Ahnood, Alastair Stacey, Kumaravelu Ganesan, Samantha G. \\ Lichter, Kate Fox, Javad Foroughi, Hamish Meffin, Gordon G. Wallace, Ray H. Baughman, Steven Prawer, \\ and David J. Garrett
}




\section{Brazing techniques for the fabrication of biocompatible carbon-based electronic devices}

Nicholas V. Apollo ${ }^{1}$, Desmond Lau ${ }^{2}$, Arman Ahnood ${ }^{1}$, Alastair Stacey ${ }^{1}$, Kumaravelu Ganesan ${ }^{1}$, Samantha G. Lichter ${ }^{1}$, Kate Fox ${ }^{3}$, Javad Foroughi ${ }^{4}$, Hamish Meffin ${ }^{5}$, Gordon G. Wallace, Ray Baughman ${ }^{6}$, Steven Prawer ${ }^{1}$, David J. Garrett ${ }^{1} *$

${ }^{1}$ : School of Physics, The University of Melbourne, Victoria 3010, Australia

2: School of Applied Sciences, RMIT

3. Centre for Additive Manufacturing, School of Engineering, RMIT University, Melbourne, Australia

${ }^{4}$ : Intelligent Polymer Research Institute, ARC Centre of Excellence for Electromaterials Science, AIIM Facility, Innovation Campus, University of Wollongong, Australia

5: National Vision Research Institute, Australian College of Optometry, Victoria 3053, and Department of Optometry and Vision Science, University of Melbourne, Victoria 3010, Australia

6. Alan G. MacDiarmid NanoTech Institute, University of Texas at Dallas, Richardson, TX 75083, USA

*Corresponding author: David Garrett; e-mail: dgarrett@unimelb.edu.au

\section{Abstract}

Translating the exceptional properties of carbon nanotubes (CNTs) and graphene into microand macrostructures has greatly broadened the potential applications of these materials. Yarns, fibers, and papers based on CNTs or graphene have been utilized as electrochemical biosensors, artificial muscles, strain sensors, and electrically-driven neural growth substrates. Prototype devices have been critical to the discovery and demonstration of the unique properties of new materials, but are not typically constructed for durability or biocompatibility because they rely on polymeric adhesives (such as conductive glues), or solders for electrical connections. In this work, we present two key metallization techniques using commerciallyavailable alloys for the integration of carbon materials with electronics: (i) laser fabrication of Au micro-circuit boards embedded in a polycrystalline diamond substrate and (ii) spotbrazing of carbonaceous fibers with Au-based pastes to enable a freestanding carbon wire with metallic (platinum or stainless steel) contacts. The carbon/metal interfaces are characterized according to their electrical properties and elemental composition at the interface. A brief review and discussion of active brazing to carbon materials is included. 


\section{Introduction}

Owing to their unique combination of biological, mechanical, electrochemical, electrical, thermal, and optical properties, carbon-based materials such as graphene, carbon nanotubes (CNTs), carbon fibers (CFs), and diamond have attracted significant scientific and industrial interest. Exploiting the properties of CNT and graphene in micro- and macroscale structures is of significant interest for engineering research and product development. ${ }^{1-3}$ For example, high strength and high conductivity fabrics based on these materials could enable a variety of applications from lightweight body armor to space elevators. ${ }^{4,5}$ Several solutions to the problem of scale have been demonstrated for both graphene and CNTs. Spinning CVD-grown aligned CNTs into a continuous yarn has enabled conductive, high-strength structures with micron diameters and macro-scale lengths. ${ }^{3}$ Control of mechanical strength and electrical/thermal conductivity of CNT yarns is established by adjusting lengths of the component CNTs, as well as the spinning angles of the fibers. ${ }^{6,7}$ Twisted and/or coiled structures of CNT yarns have been used to fabricate electrochemical as well as electrolytefree CNT torsional actuators (artificial muscles) with large-stroke and high work-capacity. ${ }^{8,9}$ Cell culture dishes containing CNT yarns have shown that neurons grow and respond to electrical stimulation delivered by the yarn. ${ }^{10}$ As bulk carriers of electricity and data, CNT yarns have been evaluated as replacements for standard $\mathrm{Cu}$ and $\mathrm{Al}$ wires due to their light weight (alleviating stress on joints), ballistic conduction (lack of scattering reduces risk of Joule heating), and capacity to handle high frequencies. ${ }^{11}$ Expanding upon the application of long-distance electricity transfer, CNT yarns have been used to build wireless data transfer networks that were mechanically-resilient and displayed frequency-independent resistive behavior. ${ }^{12}$ As a demonstration of their favorable electrochemical characteristics, CNT yarns have been used as an alternative to the standard $\mathrm{CF}$ electrochemical sensor for neurotransmitters due to an intrinsic ability to resist surface fouling. ${ }^{13}$ 
All of the aforementioned research, and much that was not mentioned, was performed using prototype devices containing silver epoxy, gold paste, solder, or mechanical clamps to create electrical connections to CNT yarns or CFs. While these connections served their respective experimental purposes, their use in fabrication does not generally yield scalable or integrated electronic devices, nor are these connection methods resilient or biocompatible enough for consideration as materials for biomedical devices. There have been several methods developed for bonding individual CNTs to metals, as well as to joining individual CNTs to other CNTs. ${ }^{14}$ However, making electrical connections to larger (micro, rather than nano) CNT composites and yarns is a different challenge. Current approaches include mechanical clamps, Ag- and Au-based epoxy adhesives, carbon solder, ultrasonic welding, and vacuum brazing. ${ }^{15,16}$ A recent, very exciting approach involves use of transition metal soldering alloys to join carbon wires using standard solder conditions (e.g., $350{ }^{\circ} \mathrm{C}$ in air). ${ }^{17}$ While it is a technique that will undoubtedly revolutionize the utility of carbonaceous wires, the $\mathrm{Cu}$ - and Sn-based alloys are unlikely to display biocompatibility due to the known cytotoxicity of these metals. ${ }^{18,19}$ We have recently developed a technique to create a hermetic diamond capsule using biocompatible gold alloys. ${ }^{20}$ Additionally, we have demonstrated a method for the construction of hermetic, biocompatible feedthroughs for a retinal prosthesis device based on conductive nitrogen-doped diamond electrodes. ${ }^{21}$ In the present paper, we extend our previous work and describe the construction of diamond-based electronic circuit substrates with embedded Ag- or Au-based interconnects and soldering pads. Finally, we describe a method to metallize the aforementioned carbonaceous wires (CNT yarns, CFs, and graphene fibers), with the option to incorporate them into the diamond circuit substrates. The methods we describe constitute an important toolkit to allow for CNTs and other similar materials to be incorporated into circuits and bonded to traditional surface-mount electronics, with special focus on biocompatible systems for implantation into the body. 


\subsection{Brazing to carbon}

Active brazing, a variation of vacuum brazing, utilizes an alloy containing an active component that reacts chemically with a relatively inert surface, such as diamond materials and ceramics. ${ }^{22,23}$ Transition metals are of particular interest due to electron vacancies in their d-orbitals, with greater numbers of vacancies leading to greater reactivity (overlap) with carbon's p-orbitals. ${ }^{24}$ In the case of carbon substrates, the active component-typically Ti, V, or $\mathrm{Cr}$ - forms a carbide interface layer that acts as a surfactant to enable the wetting of other filler metals. ${ }^{25} \mathrm{~A}$ Ag-based active brazing alloy (ABA) has been used previously to make electrical contact to a CNT bundle, and formation of the TiC interface layer was confirmed with X-ray photon scattering (XPS), though the electrical and mechanical properties were not fully characterized. ${ }^{16}$ In the present work, commercially-available ABAs were investigated for their ability to make electronic connections to several carbonaceous materials, including polycrystalline diamond (PCD), CNT yarns, graphene oxide (GO) fibers, and PAN-based carbon fibers (CFs) to enable various types of electronic devices (Figure 1).

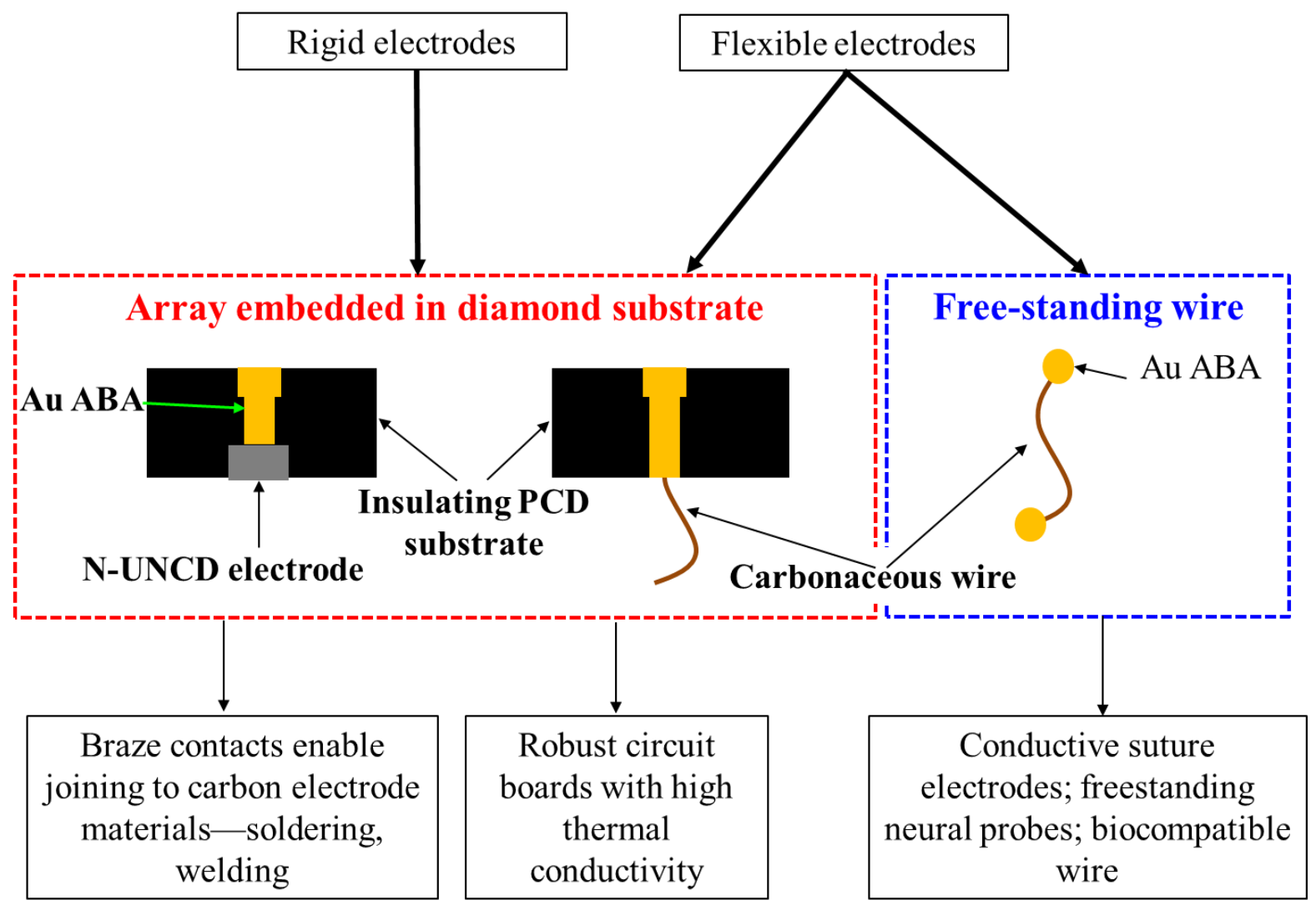


Figure 1. ABAs were used to make electrical contact to electrodes based on either conductive diamond or carbonaceous wires such as CNT yarns, CFs, or GO fibers. The option to braze either a rigid diamond or flexible carbon wire electrode into an insulating, robust substrate is the key to the realization of array-based carbon electronic devices. Another technique-a novel lift-off method - facilitates the fabrication of freestanding carbonaceous wires with metallic end-contacts. *Abbreviations in figure: $\boldsymbol{A} \boldsymbol{u} \boldsymbol{A B A}$ (Au active brazing alloy), $\boldsymbol{P C D}$ (polycrystalline diamond; electrical insulator), $\boldsymbol{N}$-UNCD (nitrogen-incorporated ultrananocrystalline diamond; electrical conductor).

In the first technique described here, ABAs were used to create embedded circuit boards in PCD, wherein the solidified ABA forms the interconnect and contact pads following mechanical polishing. The second metallization system involves making free-standing $\mathrm{Au}$ contacts to carbonaceous fibers using a graphite "lift-off" method which exploits the balance of cohesive and adhesive forces of the liquidus metal/graphite interface (Equation 1).

$$
\cos \theta=\frac{W_{A}}{\sigma_{L}}-1
$$

Where $\theta$ is the contact angle between the substrate and liquid metal, $W_{A}$ is the work of adhesion (adhesion force) between the substrate and the liquid metal, and $\underline{\sigma}_{L}$ is the cohesion force (liquid-vapour surface tension) within the liquid metal droplet. Equation 1 describes the balance of forces at the liquid metal/solid substrate interface, wherein liquid metals (intrinsically very high cohesion force materials) tend to create large contact angles on most substrates unless $\underline{\sigma}_{L}<W_{A}$, where contact angles $<90^{\circ}$ are considered “wetting”. In one facet of this work, we use active brazing to modify $W_{A}$ and create robust electrical connections to carbonaceous materials such as PCD and CFs. In another technique, we exploit the lack of adhesion and wetting between graphitic carbon and $\mathrm{Au}$, as well as the cohesion forces within the liquid metal droplet, to create a freestanding carbonaceous wire with metallic connections. Broadly speaking, the techniques described here are applicable to the development of devices in which a strong, biocompatible, and ohmic bond to carbon-based materials is required.

\section{Results and Discussion}




\subsection{Diamond circuit boards}

Three different brazing pastes were assessed for their ability to wet and bond to polycrystalline diamond (PCD) substrates (Figure 2). The elemental composition of these brazes is included in Table $\mathbf{1}$ of the Experimental section.
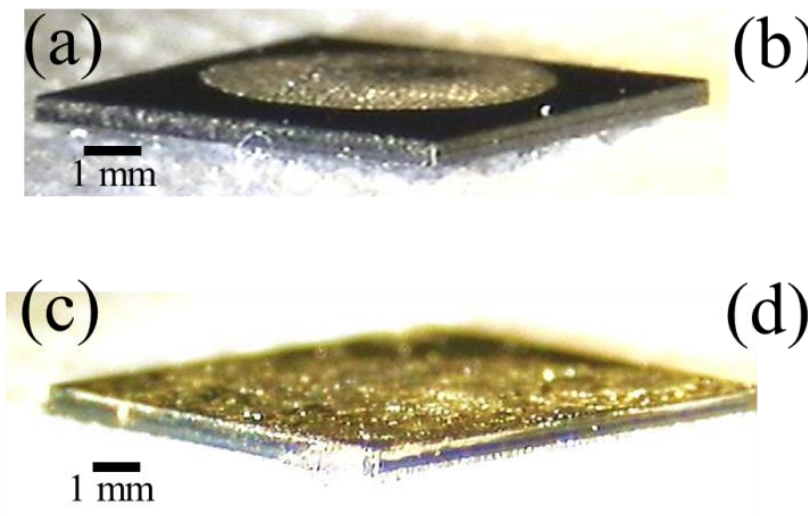

(b)
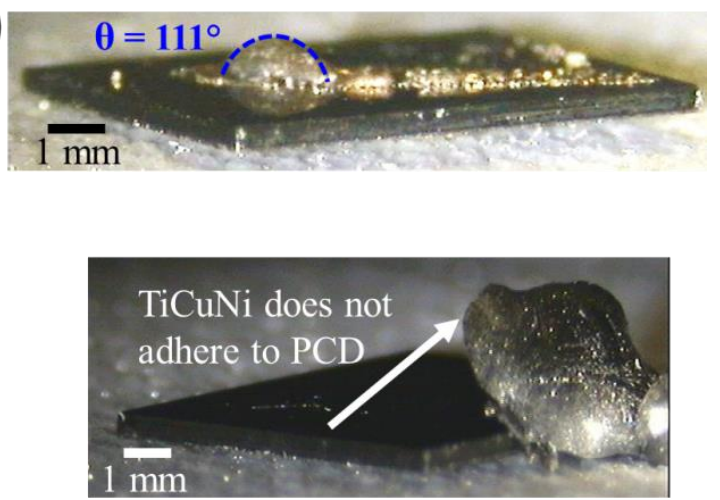

Figure 2. (a) Ag ABA wets PCD and bonds strongly. (b) Au ABA does not wet PCD, forming a droplet that makes $\sim 111^{\circ}$ contact angle with the surface; some bonding occurs but it can be removed. (c) Au ABA wets the PCD following a previous Ag ABA brazing step implemented to create an adhesion layer. (d) TiCuNi braze wets the PCD surface, but does not bond. The cooled TiCuNi droplet is easily pulled from the PCD surface with forceps.

Ag ABA was found to spread and adhere strongly to PCD, which was likely facilitated by the formation of an interfacial TiC layer (Figure 2a) (Supplementary Figure S1). Au ABA did not spread on the PCD surface upon melting and, therefore, created a spherical droplet that exhibited a $111^{\circ}$ contact angle with the surface (Figure $2 b$ ). In order to create a Au/PCD system — with the aim of biocompatibility and corrosion resistance—a Ag ABA brazing step was introduced to produce a metallic adhesion layer for a subsequent brazing step with $\mathrm{Au}$ ABA (Figure 2c). The Ag/Au two-step brazing process is illustrated in Figure 10 of the Experimental section. TiCuNi, a braze used previously for the encapsulation of medical devices, ${ }^{26}$ can wet the PCD surface upon heating but did not bond and was easily removed with forceps upon cooling (Figure 2d). Ni has been shown previously to etch diamond at low pressures during heating, in which graphitization occurs upon cooling. ${ }^{27}$ It is possible that this graphitic layer is preventing the spreading of $\mathrm{Au}-\mathrm{ABA}$, as well as the lack of adhesion of the 
TiCuNi upon cooling. The effect is exaggerated with TiCuNi due to a 5-fold increase in $\mathrm{Ni}$ content compared with Au ABA (Table 1). If a metal is unable to wet or bond to PCD, it is not suitable for the creation of embedded, thick film conductors or contact pads required for the embedded circuit structures presented here.

A $532 \mathrm{~nm} \mathrm{Nd:YAG} \mathrm{laser} \mathrm{was} \mathrm{used} \mathrm{to} \mathrm{create} \mathrm{microscopic} \mathrm{grooves} \mathrm{in} \mathrm{a} \mathrm{PCD} \mathrm{substrate} \mathrm{prior} \mathrm{to}$ brazing and mechanical polishing processes. This process is illustrated in Figure 9 of the Experimental section. The laser-cut microstructures and resultant brazed/polished devices were imaged using optical microscopy, SEM, and Micro-CT (Figure 3). Figure 3a shows two magnifications of microstructures milled into PCD. The interconnect widths achieved so far with this technique range between 6-10 $\mu \mathrm{m}$. A correlation between laser power and groove width was found using SEM imaging (Supplementary Figure S2). Smaller features are likely possible following more extensive laser parameter optimization. Only Ag/PCD and $\mathrm{Au} / \mathrm{PCD}$ (with Ag-ABA adhesion layer) devices were fabricated due to the poor adhesion of TiCuNi braze and the poor wetting of pure Au ABA on PCD substrates. Ag-ABA spreads and flows during heating to fill in laser-milled circuit structures, which demonstrates a significant advantage over conventional "line-of-sight" thin film metallization procedures. A Micro-CT image (Figure 3c) demonstrates the complex three-dimensional assembly of braze wires and contact pads utilized to contact conductive diamond electrodes (dotted circle) in a full electrode array device. The resistance of Au microwires embedded in PCD was measured using a transmission line set-up to correct for contact resistance. The relationship between the resistance of a $500 \mu \mathrm{m}$ length Au interconnect and laser power utilized to mill the track is shown in Figure 3d. 


\section{(a)}
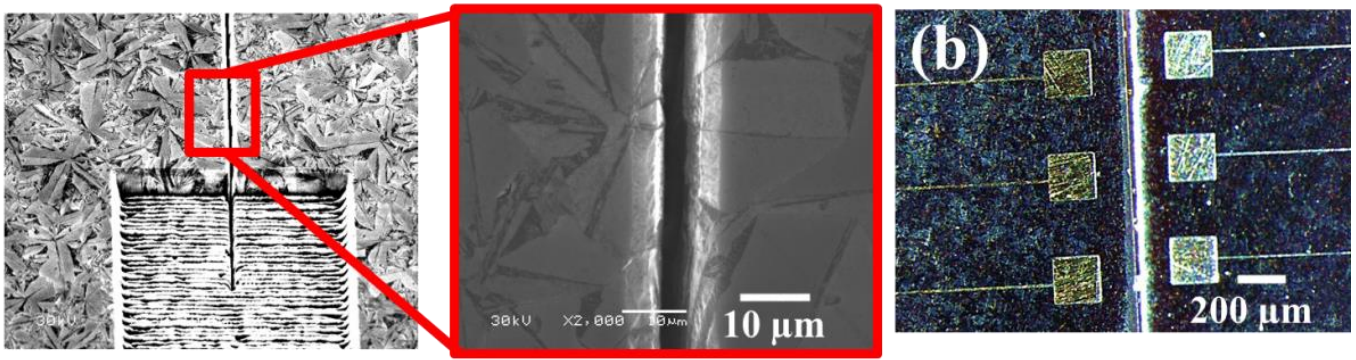

(c)

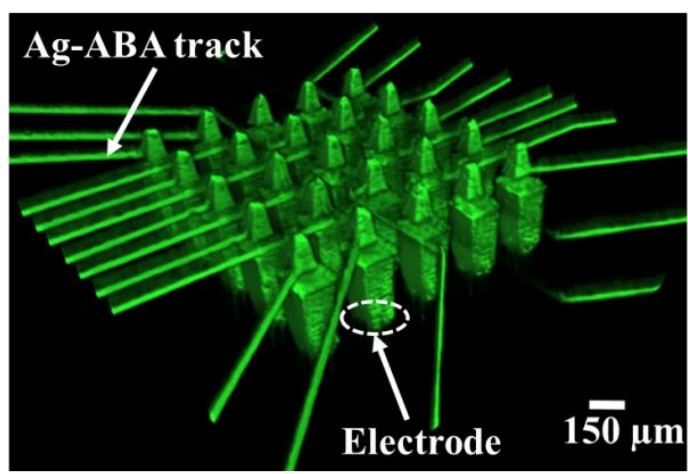

(d)

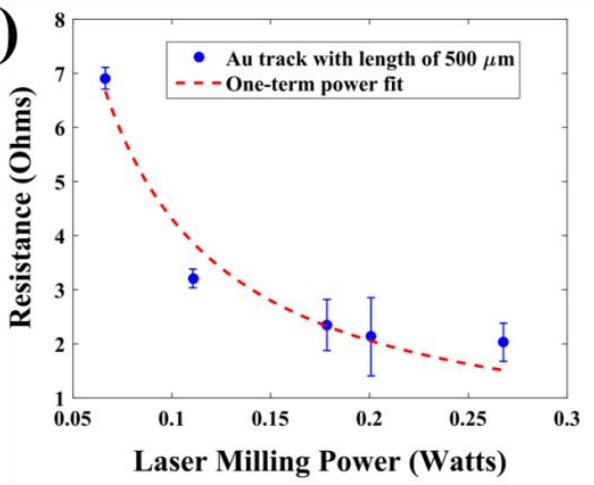

Figure 3. Embedded circuit boards produced with laser engraving, active brazing, and mechanical polishing. (a) SEM image of a laser-cut interconnect (right) and contact pad. (b) $\mathrm{Au}$ (left) and Ag (right) circuit structures after mechanical polishing. (c) A microcomputed tomography (micro-CT) image of the metal interconnects embedded in a diamond substrate. Note: the diamond is highly transparent to the CT X-rays. (d) Relationship between laser power used to mill the circuit structure and resistance of the embedded track. The length of the track $(n=3)$ measured at each power was $500 \mu \mathrm{m}$ and was made using the 2-step brazing process: Ag ABA, followed by Au.

The 3D nature of the interconnect leads to a significant decrease in electrical resistance compared with a thin film metal contact according to $R=\rho L / A$, where ' $R$ ' is resistance, ' $\rho$ ' is material resistivity, ' $\mathrm{L}$ ' is interconnect length, and ' $\mathrm{A}$ ' is interconnect cross-sectional area. The polished circuit boards are durable and ideal for thermal management applications due to diamond's extremely high thermal conductivity $(1-2 \mathrm{~kW} / \mathrm{mK}) .{ }^{28}$ The workability of both Auand $\mathrm{Ag}-\mathrm{ABA}$ interconnects is an important consideration. Both varieties of braze are solderable and easily laser welded to, enabling compatibility with traditional surface-mount technology (Supplementary Figure S3). Brazing individual CFs into a diamond substrate was achieved using a screen-printing technique to avoid the need for mechanical polishing

\section{(Figure 4).}




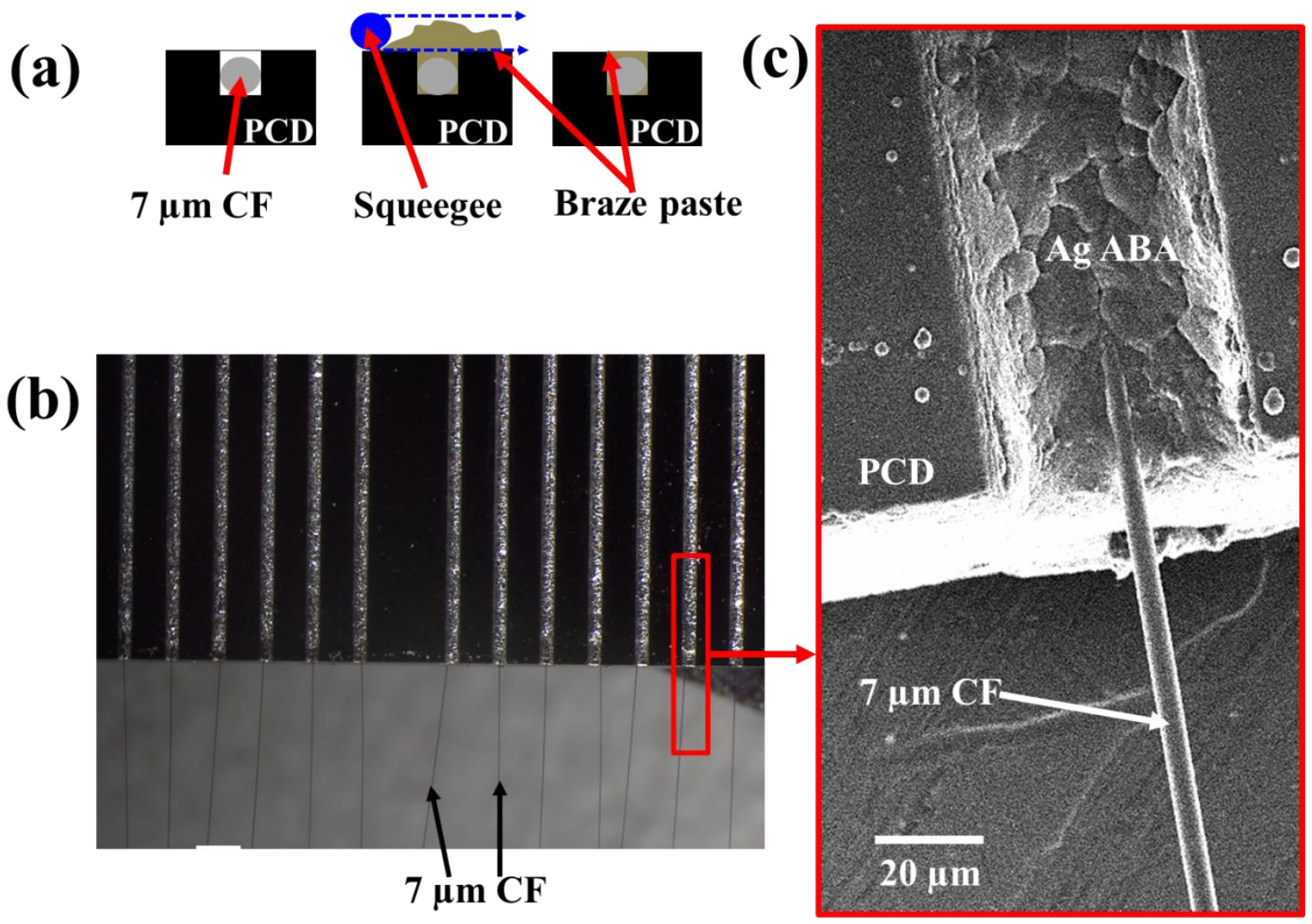

Figure 4. (a) Individual $7 \mu \mathrm{m}$ CFs were manually-placed into laser-cut grooves of the PCD substrate and secured in place with braze paste. Due to the presence of laser-cut grooves, excess paste was removed by wiping with a squeegee to avoid a mechanical polishing step. The substrates were heated to melt the Ag ABA paste, forming an embedded electrode and metallic interconnect in a PCD substrate $(b, c)$.

Avoiding mechanical polishing is important for delicate, small, and fragile electrode materials.

However, because the brazing pastes are comprised of both metal beads and polymeric binder, the functional volume of the interconnect is reduced upon melting, leading to some loss in conductivity in comparison with a thicker interconnect. We are currently investigating methods for protecting the CFs, and other carbonaceous wires, during mechanical polishing to enable more complex, robust device architectures.

\subsection{Metallization of free-standing carbon wires}

If the carbonaceous wire is to be used as a free-standing electrochemical probe or conduction line, a "lift-off" method is required. Of the three varieties of ABA paste examined, Au ABA is 
preferred because it does not form a strong bond to the graphite substrate following a melting cycle, so the entire wire can be lifted off of the substrate with the contacts intact, preventing damage to the wire. Both $\mathrm{Ag} \mathrm{ABA}$ and TiCuNi bonded strongly to the graphite substrate preventing facile lift-off and sometimes leading to mechanical damage of the fiber. The enhanced bonding of the TiCuNi/graphite system in comparison with the TiCuNi/PCD system is possibly explained by graphite's porosity, which creates a higher contact surface area compared with diamond (reference). Au does not bond to the graphite due to the known immiscibility of graphitic carbon and $\mathrm{Au}$ (brazing reference). When the graphite substrate was heated, the Au-ABA formed a spherical metallic contact, enclosing both the carbonaceous fiber and a metallic wire (if desired) within it (Figure 5).
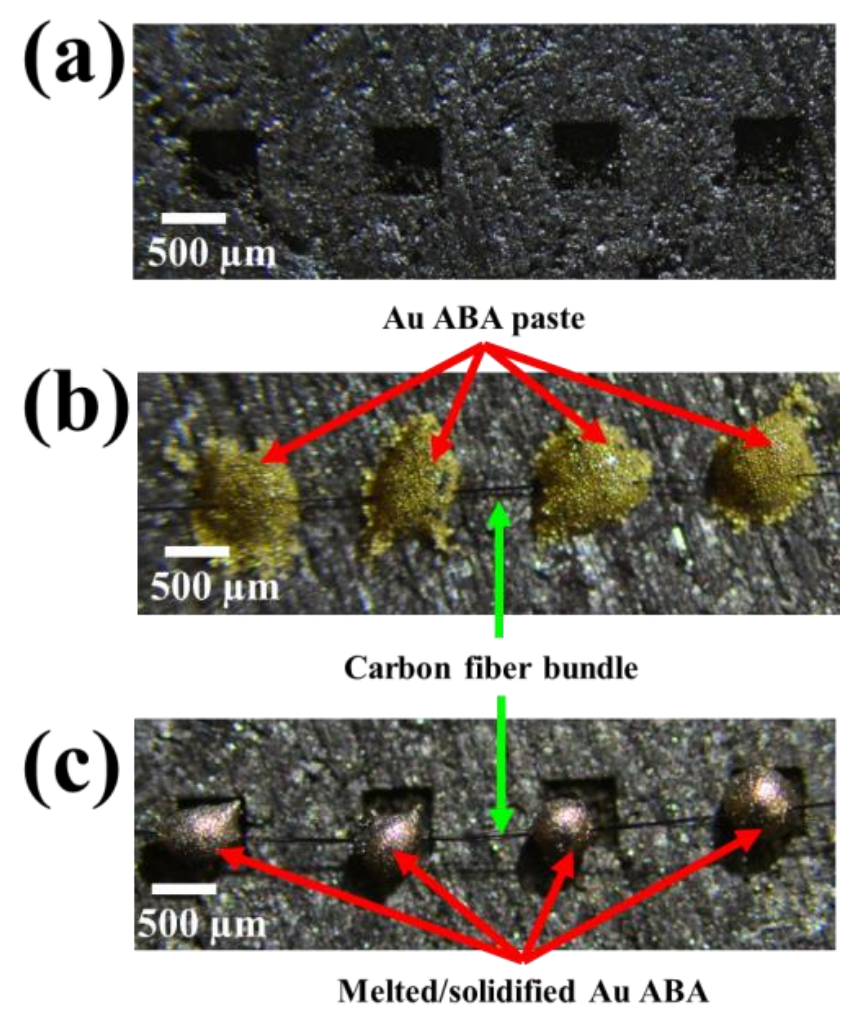

Figure 5. Graphite lift-off method demonstrating resolution of spot-brazing free-standing carbon wires. (a) Graphite wafer laser milled for spot brazing technique. (b) Au ABA paste placed over grooves and (c) solidified Au contacts on carbon fiber bundle. The intact device is easily removed from the graphite wafer due to the lack of chemical bonding between $\mathrm{Au}$ and graphite. 
Additionally, both TiCuNi and Ag-ABA wetted portions of the carbon wire, likely changing its mechanical and structural properties, limiting the resolution of the contact area

(Supplementary Figure S4). This technique is a potential improvement over mechanical clamps, especially considering the diameters of the fibers studied here $(7-50 \mu \mathrm{m})$ because mechanical stress from clamps would likely fray or damage the fibers. Voltage sweeps (-1 to $+1 \mathrm{~V})$ were performed while current was recorded across the PtIr/CNT yarn (Figure 6b), suggesting a linear (non-rectifying) contact in the tested voltage range. The resistivity, $\rho$, of the CNT yarns in this work was comparable to those, of similar size and length, measured by Jasinghe et al. ${ }^{29}$ We measure an average $\rho$ of $4.3 \times 10^{-3} \Omega \cdot \mathrm{cm}$ for the PtIr/Au/CNT yarn structures and Jasinghe et al report a $\rho$ of $1.7-3 \times 10^{-3} \Omega \cdot \mathrm{cm}$ for pristine CNT yarns. Further investigation is needed to estimate the contact resistance of this system.
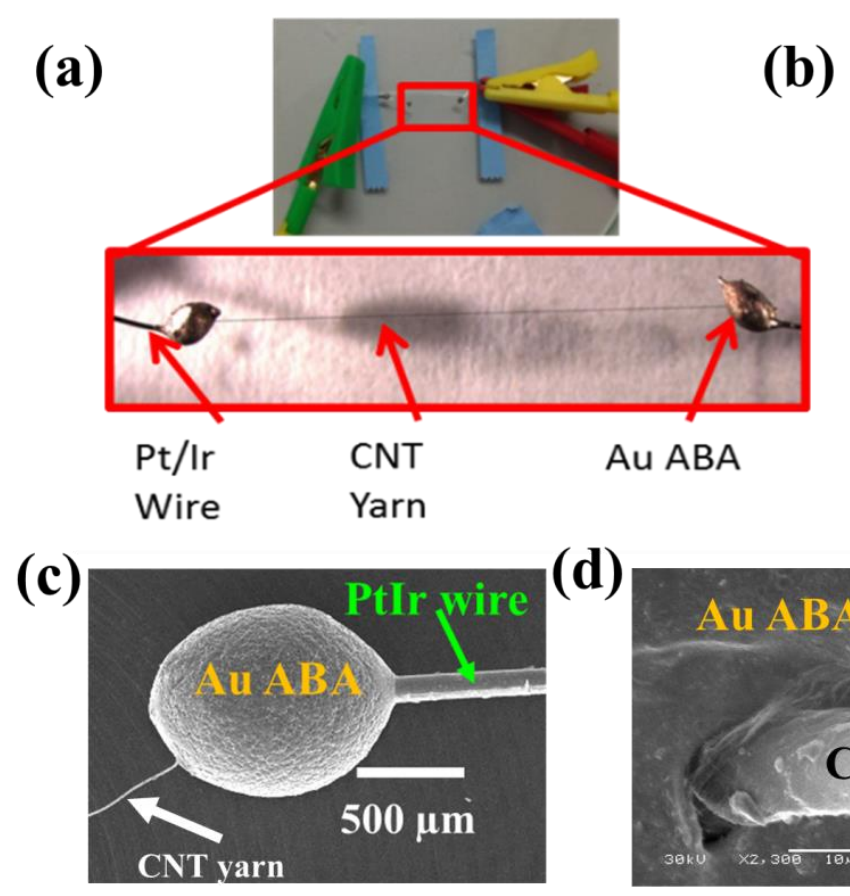

(d)

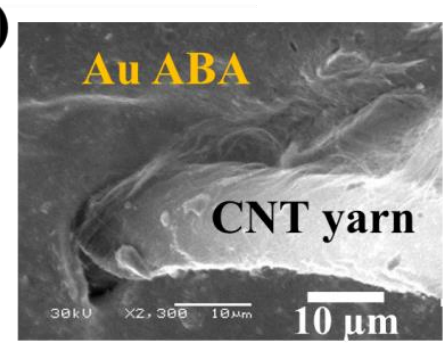

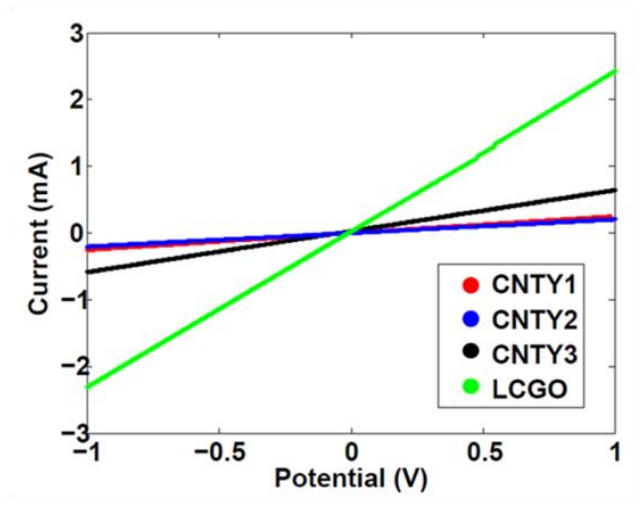

(e)

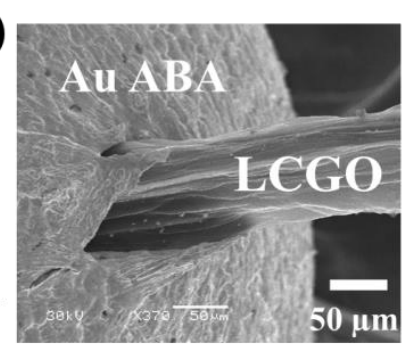

Figure 6. Electrical characterization and SEM imaging of free-standing carbonaceous wires with Pt wire contacts. (a) Optical photograph of PtIr/Au/CNT yarn system during electrical measurement. (b) Linear (Ohmic) I-V curves of several CNT yarns and one graphene oxide (LCGO) fiber sample. (c) SEM image of the Au contact between the PtIr wire and the CNT yarn. (d) 2300X magnification of the junction between the Au contact and the yarn. (e) 370X magnification of the Au contact on LCGO fiber. 


\subsection{Material characterization}

\subsubsection{Pre- and post-brazing Raman spectroscopy}

The structures described above were characterized using 514 nm Raman spectroscopy (inVia) to investigate structural changes in the materials before and after high temperature brazing

(Figure 7). The Au ABA samples were chosen for investigation because they were subject to the highest melting ranges $\left(1003-1030{ }^{\circ} \mathrm{C}\right)$.
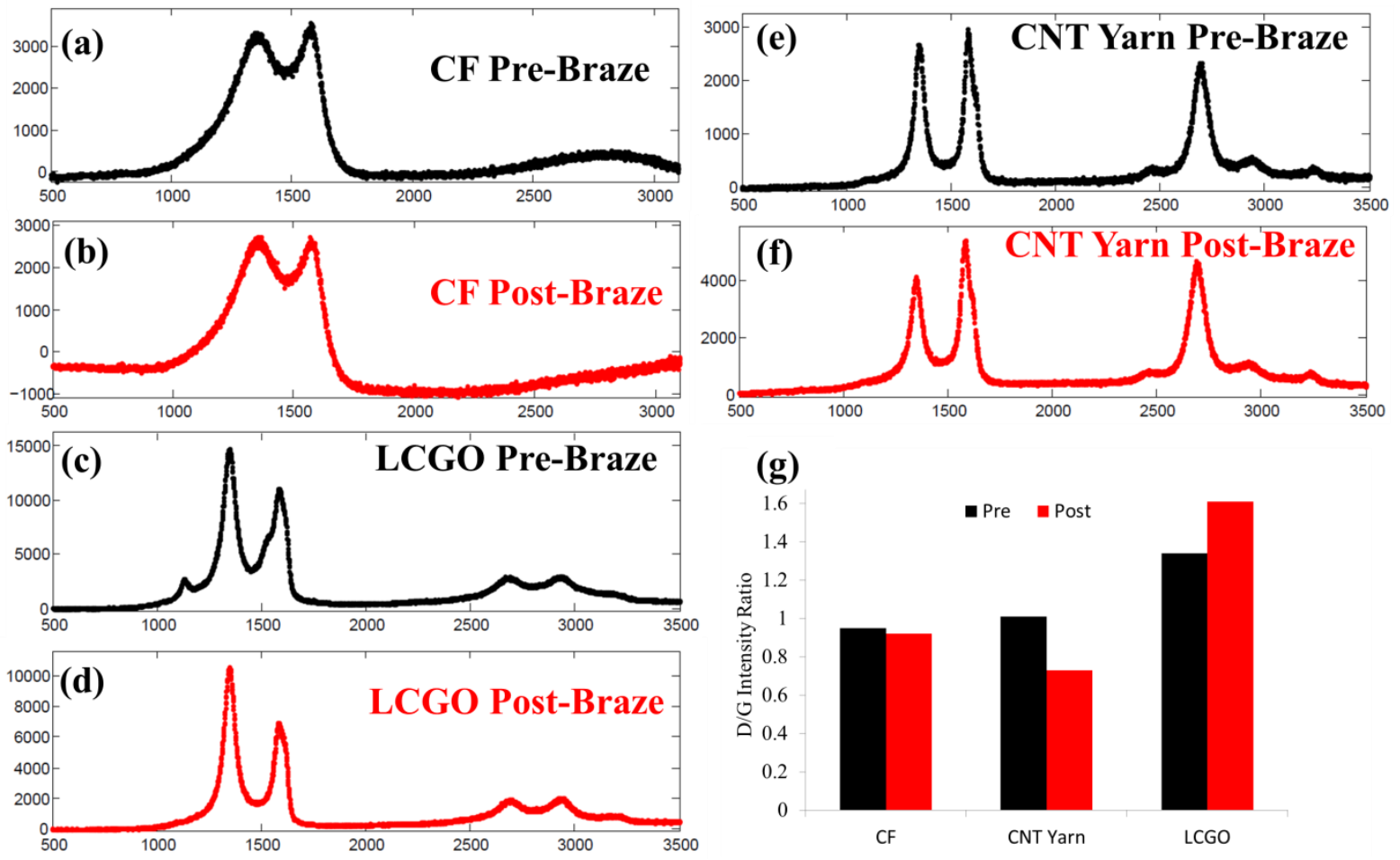

Figure 7. Raman spectroscopy of CNT yarns (a,b), LCGO fibers (c,d), and CF bundles (e,f) before and after brazing. (g) Chart illustrating change in D/G intensity with brazing. The Dpeak (or disorder peak) is located around $1330-1350 \mathrm{~cm}^{-1}$ and the G-peak (or graphite band) is located around $1580-1590 \mathrm{~cm}^{-1}$.

In an attempt to improve the thermal and electrical properties of CNTs and composites, gamma irradiation and high temperature treatments have been previously investigated. ${ }^{30}$ The CNT yarn spectra suggest a decrease in the intensity ratio of D/G peaks following brazing, 
suggesting the decrease of "disorder" in the lattice (Figure 7g). A similar result was observed by Pieoli et al. ${ }^{30}$ It of interest that creating brazed contacts at either end of a woven or spun material, such as CNT yarn, prevents unwinding and generally helps to keep the fiber intact. Mechanical clamps, which apply large forces to the end of the yarn, can lead to fraying and damage, especially at smaller yarn diameters. ${ }^{15}$ The before and after Raman spectra of CFs reveals very little change in the microstructure, aside from the loss of a broad peak at around $2750 \mathrm{~cm}^{-1}$ following brazing (Figure 7a,b). This peak, known as $\mathrm{G}^{\prime}$, is described as an overtone of the D-peak. ${ }^{31}$ The presence of a prominent D-peak in CFs based on polyacrylonitrile (PAN) is typically attributed to poor graphitization and is never present in pure crystallized graphite. ${ }^{31}$ The G' peak appears quite prominent in both CF spectra presented here (Figure 7a,b), though there appears to have been some moderate increase in graphitization following brazing. Additionally, the loss of the D-peak overtone (the G' peak) potentially suggests the increased crystallinity of the material due to increased graphitization. However, it is important to note that the graphitization process during commercial CF manufacturing occurs at around $2600{ }^{\circ} \mathrm{C}$ - much higher than is used to create Au contacts in this study. ${ }^{31}$ The Raman spectra of the LCGO fiber revealed several peaks typically seen in the spectra of graphite, carbon fibers, and graphene oxide (Figure 7c,d). Some major changes occurring following brazing include: (1) loss of a peak at $\sim 1100 \mathrm{~cm}^{-1}$, (2) increase in D/G intensity ratio, and (3) loss of a "shoulder" on the G' peak. It is possible that the loss of these peaks is the result of the loss of a surface functionality as a result of high temperature treatment, such as a C-O group. However, further investigation is required to acquire a full understanding of the structural changes occurring not only as a result of the high temperature processes in the presence of various metals.

\subsubsection{Braze/carbon interface characterization}


Au ABA formed a seal around the bundle, but had minimal reaction with the graphite

substrate, enabling the fiber/ABA structure to be easily lifted from the substrate. However, if enough force is applied, the Au contact can be removed from the fiber. To investigate the interface between the fibers and the brazing alloys, the same samples described above were molded, polished and examined using energy dispersive X-ray spectroscopy (EDX) and SEM (Figure 8). All analysis was performed on the cross-section of the fiber-metal interface as illustrated in Figure 8a.

(a)
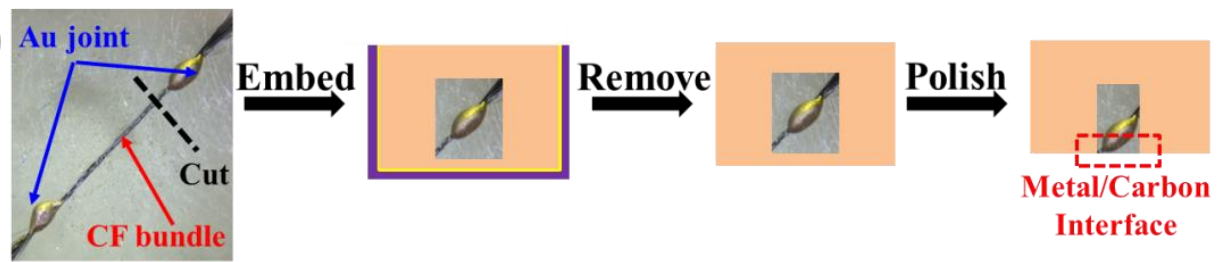

(b)
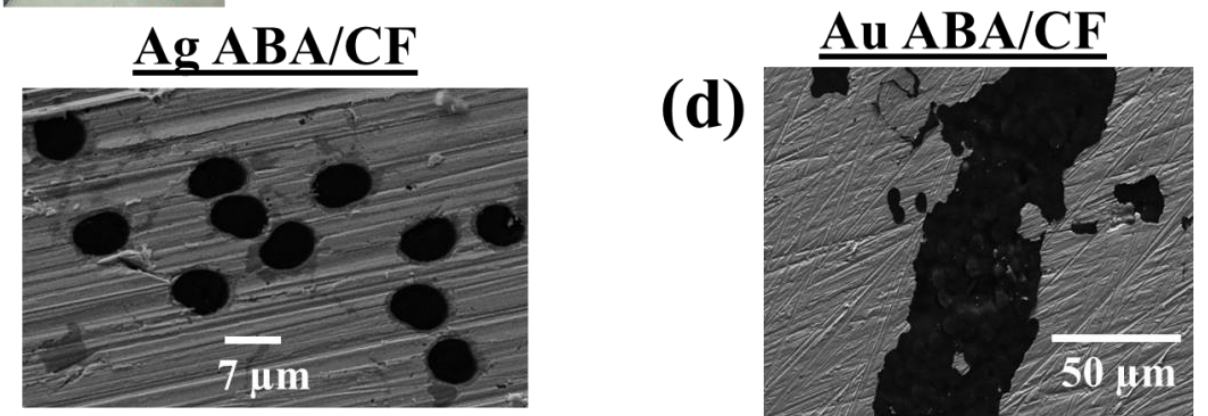

(c)
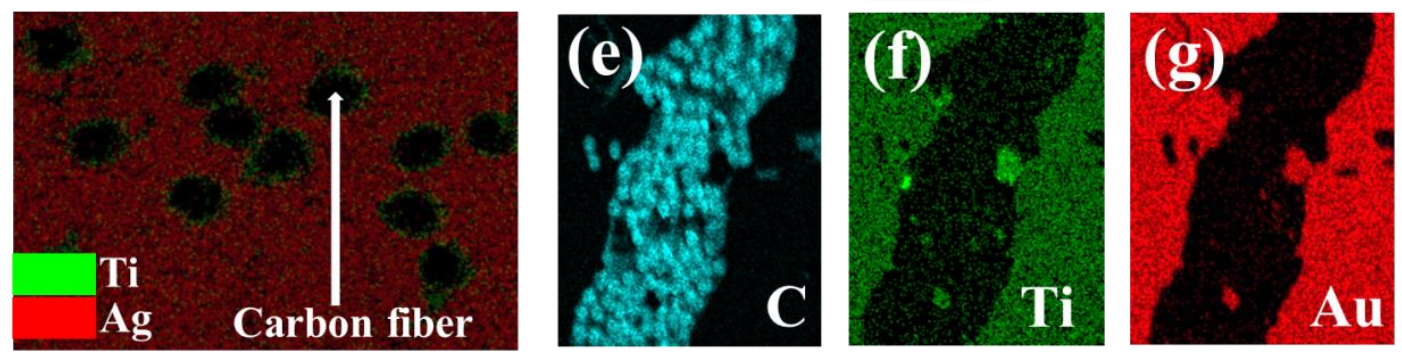

Figure 8. (a) Process for preparing interface examination sample. A silicone rubber mold (purple outline) was cast using a $10 \mathrm{~mm}$ steel cylindrical plug. Next, the CF/ABA samples were cut close to to the joint and placed into the mold with super glue until fixed. Araldite epoxy resin was poured in to the mold, covering the entire sample, which was then polished and examined with EDX and SEM. (b) Ag ABA surrounds individual $7 \mu \mathrm{m}$ diameter CFs. (c) EDX reveals that Ti (green) has migrated to the fibers, forming TiC that enables wetting of $\mathrm{Ag}$ (red). (d) Au ABA, rather than reacting with individual fibers, forces the individual fibers together to form a bundle (e), with only small amounts of Ti diffusing in (e).

The Ag-ABA/CF interface revealed separation of the $\mathrm{CF}$ bundle into individual $7 \mu \mathrm{m}$ fibers

(Figure 8b). EDX of the interface revealed rings of Ti around the fibers and intimate contact 
with Ag, perhaps suggesting a TiC reaction product (Figure 8c). Due to the presence of the $\mathrm{TiC}$ surfactant layer, an intimate bond with $\mathrm{Ag}$ is made possible. Au-ABA reacted quite differently with the CF bundle. Rather than splaying the bundle, the cohesion forces within the liquidus metal enclosed the fiber bundle (Figure 8d). There was some Ti present in the joint, but not selectively around the $\mathrm{CF}$ as observed in the Ag-ABA sample. This difference in activity can be explained by the fact that $\mathrm{Au}$ does not wet $\mathrm{TiC}$ due to the precipitation of a graphite layer. ${ }^{32}$ At the interface of the $\mathrm{Au} / \mathrm{TiC}$ system, there is an important reaction to consider:

$$
T i C \rightarrow T i+C_{g}
$$

Where $\mathrm{C}_{\mathrm{g}}$ is a graphitic carbon precipitate. Adding between $3.7 \%$ and $7.5 \% \mathrm{Ni}$ (or 7.1 to $15.8 \% \mathrm{Fe}$ ) to the $\mathrm{Au}$ alloy can dramatically reduce the contact angle between $\mathrm{Au}$ and a $\mathrm{TiC}$ surface. ${ }^{33,34}$ Another option to improve Au wetting to $\mathrm{TiC}$ is to introduce $\mathrm{Cu}$, though the effect is not as prominent as with the $\mathrm{Au} / \mathrm{Ni}$ or $\mathrm{Au} / \mathrm{Fe}$ alloys. In previous literature describing $\mathrm{Au} / \mathrm{Cu}$ experiments, it was hypothesized that $\mathrm{Cu}$ would reduce the reactivity of the $\mathrm{Au}$, thereby reducing the amount of $\mathrm{Ti}$ pulled from $\mathrm{TiC}$ and ultimately reducing the amount of graphitic carbon precipitation $\left(\mathrm{C}_{\mathrm{g}}\right) \cdot{ }^{32,33}$ However, the addition of $\mathrm{Cu}$ could potentially compromise biocompatibility. Titrating Au alloy compositions to enhance carbon bonding without cytotoxic consequences warrants further investigation.

\section{Conclusions}

Two key methods for fabricating electronic microdevices with PCD and carbon-based wires (CNT yarns, CFs, and LCGO fibers) have been described, with focus on biocompatibility of all components. For PCD electronics, Ag ABA is used due to its wetting and covalent bonding to diamond via reactive formation of TiC. Au ABA can be used following creation of an Ag ABA interfacial layer, as it does not wet PCD. Both Au and Ag contact pads of the 
embedded PCD circuit boards are compatible with commercially-available solders and are easily laser-welded for solder-free joining. The non-wetting method of enclosing carbonaceous fibers and yarns was used to create strong, low-resistance, and Ohmic connections between PtIr wires and carbonaceous fibers. Structural and interfacial examinations of these structures lend insight into the forces at play during both the wetting and non-wetting fabrication of carbonaceous devices. We exploit surface tension effects of two commercially-available active brazing alloys to create two different device architectures: (1) The wetting technique was used, in conjunction with mechanical polishing, to create robust circuit substrates with embedded $\mathrm{Ag}$ or $\mathrm{Au}$ interconnects and contact pads. The option to braze a variety of carbonaceous wires into the substrate illustrates utility of the method for enabling all-carbon microwire electrode arrays.

(2) The non-wetting technique was developed to enable freestanding carbon wires with $\mathrm{Au}$ or $\mathrm{Au} / \mathrm{PtIr}$ wire contacts. This technique is interesting for the creation of not only free-standing carbonacoues conductors, but also for neural probes or sensors for both central and peripheral nervous system integration. We have previously described methods to fabricate neural stimulation and recording electrodes from carbonaceous wires, demonstrating both in vitro and in vivo functionality. ${ }^{35}$

\section{Experimental}

Wetting of PCD by braze alloys

Thermal grade polycrystalline diamond (PCD) substrates (TM-250, Element Six) with a thickness of $250 \mu \mathrm{m}$ were laser-cut into $5 \times 5 \mathrm{~mm}$ squares. Approximately $6.5 \mathrm{mg}$ of either TiCuNi, Ag ABA, or Au ABA paste were placed ontop of the PCD substrate. Samples were 
placed in a brazing furnace under vacuum heated until the braze was visibly melted, then held at temperature for 1 minute. Samples were cooled and imaged using an optical microscope. The contact angle of $\mathrm{Au} \mathrm{ABA}$ on PCD was measured using the low-bond axisymmetric drop shape analysis in the Droplet-Analysis package in ImageJ image processing software. ${ }^{36,37}$

\section{Fabrication of PCD circuit substrates}

Thermal grade PCD substrates (TM-500, Element Six) with a thickness of $500 \mu \mathrm{m}$ were laser engraved using a $532 \mathrm{~nm} \mathrm{Nd:YAG} \mathrm{laser} \mathrm{(Oxford} \mathrm{lasers)} \mathrm{at} \mathrm{a} \mathrm{pulse} \mathrm{frequency} \mathrm{of} 10 \mathrm{kHz}$. A boiling solution of sodium nitrate and sulfuric acid was used to remove debris from the engraved pattern, followed by 5 minutes of ultrasonication in acetone. The PCD substrates were rinsed with isopropanol and deionized water, then dried with nitrogen. Groove width was correlated with laser power using scanning electron microscopy (SEM) images. The contents and properties of the ABA pastes studied are listed in Table 1.

\begin{tabular}{cccccccc}
$\begin{array}{c}\mathrm{ABA} \\
(\text { Manufacturer })\end{array}$ & $\mathrm{Ag}(\%)$ & $\mathrm{Au}(\%)$ & $\mathrm{Cu}(\%)$ & $\mathrm{Al}(\%)$ & $\mathrm{Ti}(\%)$ & $\mathrm{Ni}(\%)$ & $\mathrm{MP}\left({ }^{\circ} \mathrm{C}\right)$ \\
\hline $\begin{array}{c}\text { Ag-ABA } \\
\text { (Wesgo) }\end{array}$ & 92.75 & - & 5 & 1 & 1.25 & - & 912 \\
$\begin{array}{c}\text { Au-ABA } \\
\text { (Morgan) }\end{array}$ & - & 96.4 & - & - & 0.6 & 3 & 1003 \\
$\begin{array}{c}\text { TiCuNi } \\
\text { (Wesgo) }\end{array}$ & - & - & 15 & - & 70 & 15 & 960 \\
\hline
\end{tabular}

Table 1. Manufactuer, elemental composition, and melting point (MP) of the ABA pastes studied.

ABA paste was spread over the PCD substrate. Samples were transferred to a $10 \times 10 \mathrm{~mm}$ graphite wafer, placed inside an electron beam deposition system (Thermionics) and evacuated to $1 \times 10^{-6} \mathrm{~Pa}$. The electron beam was focused onto the graphite substrate, rather than the diamond sample, and current was increased until both the graphite and PCD were 
glowing red, ultimately leading to the ABA melting and spreading over the substrate and into the laser-milled patterns. Current was decreased over the course of 1 minute and the samples were cooled over the course of 1 hour in a $\mathrm{N}_{2}$ environment. The brazed samples were removed from the deposition chamber and mechanical polishing was performed with a diamond-embedded polishing plate (Struers Inc.), using deionized water as a coolant. The samples were polished until embedded Ag ABA or Au ABA tracks were revealed and residual metals between patterns removed, ensuring no shorting between interconnects. Resistance between isolated interconnects was measured to ensure no shorting (e.g., $>\mathrm{M} \Omega$ resistance) between unconnected tracks. If shorting did occur, due to residual unpolished metal, mechanical polishing was continued until it was removed. Fiber or wire electrodes can be incorporated into laser cut grooves, followed by "screen-printing" ABA paste into the grooves holding them securely during sample transfer. Screen-printing removes the need for a final mechanical polishing step which can damage the very sensitive fibers. The fabrication of $\mathrm{Au} / \mathrm{PCD}$ circuit boards requires an additional brazing step to metallize the circuit substrate

\section{(Figure 9).}

(a)

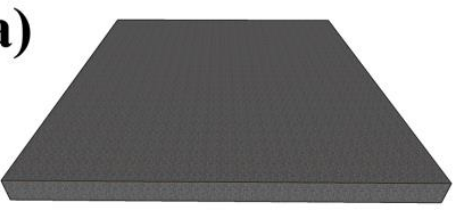

(d)

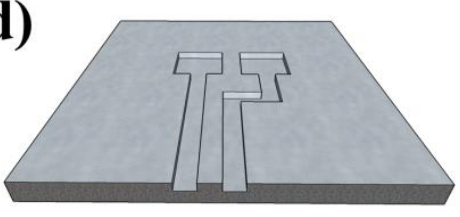

(g)

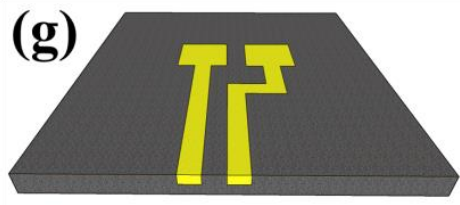

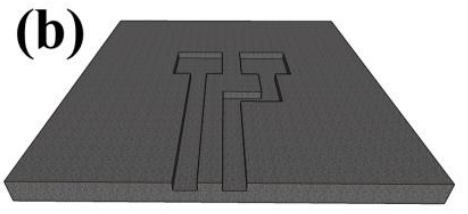

(e)

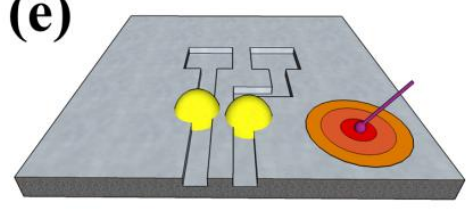

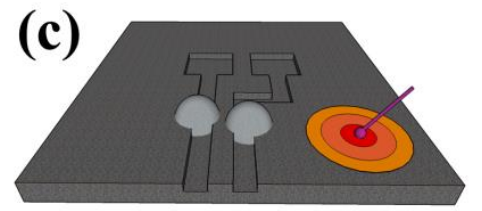

(f)

(h)

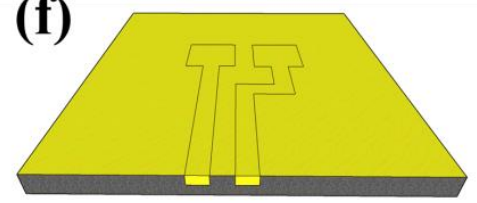

Figure 9. An extension of the method described in Figure 2. (a-b), circuit patterns were laser cut into the diamond substrate and the surface treated with a combination of sulfuric acid and sodium nitrate. (c) Ag-ABA paste was applied to the surface melted in an electron beam 
deposition system. (d) The electron beam was continuously applied until a majority of Ag evaporated from the surface, leaving behind a conformal film for Au adhesion (e) A Au-based paste was applied to the surface and melted with the aforementioned method (f). (g) The substrate was mechanically-polished, revealing Au circuit structures. (h) Finally, connections or components were attached with laser welding, soldering, or wirebonding if necessary.

\section{Carbon wire preparation}

MWNT forest was synthesized by catalytic chemical vapor deposition using acetylene gas as the carbon source. Carbon nanotubes in the 400 um tall forests typically had diameters of 10 $\mathrm{nm}$. The CNT yarns were drawn from the forest by pulling and twisting as described by Zhang et al. ${ }^{38}$ Graphene oxide (GO) was prepared from intercalated graphene flakes. The liquid crystallite state of graphene oxide was used to wet-spin conductive fibers as described previously. $^{39}$

\section{Metallization of freestanding carbon wires}

On a graphite substrate, two mounds of ABA paste were placed on opposite sides of a $10 \times 10$ mm graphite wafer. Either a bundle of CFs (Goodfellow), a single CNT yarn (15 $\mu \mathrm{m})$, or a graphene oxide fiber $(50 \mu \mathrm{m})$ was placed across the mounds and pressed into them.

Additionally, one PtIr wire $(75 \mu \mathrm{m}$, Goodfellow) was placed into each of the ABA paste mounds. The graphite substrate was transferred to an electron beam deposition system (Thermionics) and evacuated to $1 \times 10^{-6} \mathrm{~Pa}$. The electron beam was focused onto the graphite substrate — as described above for PCD circuit board construction — and the current was increased until the substrate was glowing red. This process is illustrated in Figure 10. 
(a)

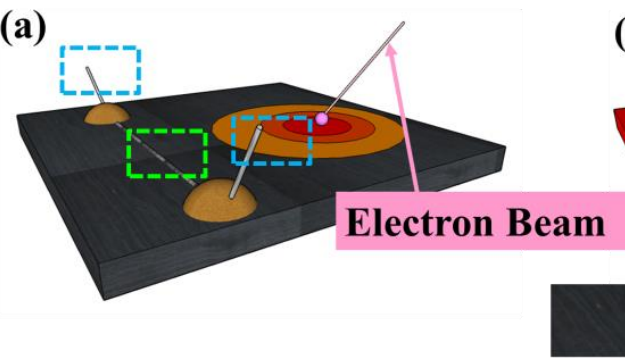

(c)

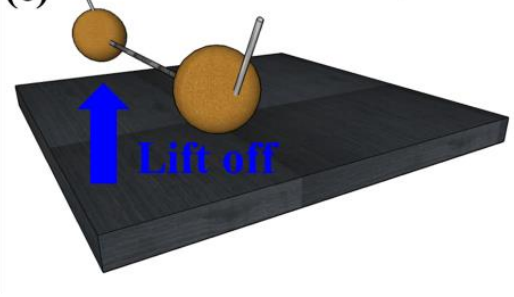

(b)

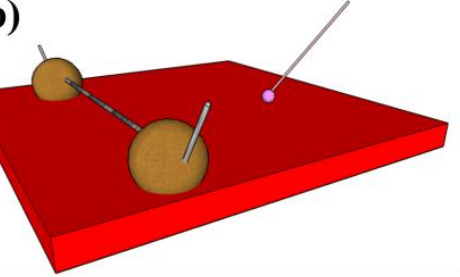

Graphite substrate

Graphite substrate (heated)

ABA paste

Carbonaceous wire

Platinum wire

Figure 10. (a) Fabrication of freestanding metal/ABA/CNT yarn wires. Mounds of ABA paste were placed onto a graphite substrate and either CNT yarns, CFs, or graphene oxide fibers were pressed into them. PtIr wires were pressed into the end for testing.

Once melting of the ABA was observed, the current was ramped down over 1 minute and the samples were cooled for 30 minutes in an $\mathrm{N}_{2}$ environment. Au-ABA samples were easily removed from the graphite substrate due to lack of reaction, while considerable force was needed to remove the $\mathrm{Ag} \mathrm{ABA}$ and TiCuNi samples.

\section{Electrical characterization}

The resistance of the Au ABA interconnects embedded in the polished PCD substrates were measured using a 2-way probe station and a multimeter (Keithley Instruments). Transmission line devices were fabricated to correct for contact resistance. The electrical behavior of the freestanding PtIr/Au/CNT yarn structures was characterized by performing voltage sweeps (-1 to +1 Volt) with a potentiostat (eDAQ) while measuring current.

\section{Material and interface characterization}


Raman spectroscopy (inVia Raman, Renishaw) was used to investigate the effects of high temperature brazing on the microstructure of CFs, CNT yarns, and reduced graphene oxide fiber (LCGO). A $514 \mathrm{~nm}$ green laser with a power of 50\% (15 mW) was used for CF bundles, while a power of $5 \%(1.5 \mathrm{~mW})$ was used for $15 \mu \mathrm{m}$ CNT yarns and $40 \mu \mathrm{m}$ LCGO to avoid ablation damage. Samples were scanned before and after high temperature brazing (1003$\left.1030^{\circ} \mathrm{C}\right)$. The laser was focused on the center of the sample and an exposure time of 60 seconds was used. The acquired signals were processed in Matlab using a linear baseline correction procedure, followed by Gaussian fitting to resolve peaks. The metal/fiber interfaces were studied by embedding brazed samples in epoxy resin (Araldite) and mechanicallypolishing them to reveal the cross-section. SEM images were acquired and energy-dispersive X-ray spectroscopy (EDX) was used to identify elemental composition at the interface of both $\mathrm{Ag}-\mathrm{ABA} / \mathrm{CF}$ and $\mathrm{Au}-\mathrm{ABA} / \mathrm{CF}$ joints.

\section{Acknowledgements}

Many thanks to David Thomas for assistance with Micro-CT imaging and Owen Burns for insightful technical conversations. NVA is supported by a MMI-CSIRO Material Science PhD Scholarship. DJG is supported by ARC DECRA grant DE130100922.This research was supported by the Australian Research Council (ARC) through its Special Research Initiative (SRI) in Bionic Vision Science and Technology grant to Bionic Vision Australia (BVA). JF is supported by the Australian Research Council under Discovery Early Career Researcher award (Javad Foroughi DE12010517). GGW acknowledges ARC Laureate Fellowship and support through the ARC Centre of Excellence for Electromaterials Science. 
Supporting Information Available: Figures S1-S4. This material is available free of charge via the internet at http://pubs.acs.org.

\section{References}

1. Li, Y.-L., Kinloch, I. \& Windle, A. Direct Spinning of Carbon Nanotube Fibers from Chemical Vapor Deposition Synthesis. Science 304, 276-278 (2004).

2. Jiang, K. et al. Superaligned Carbon Nanotube Arrays, Films, and Yarns: A Road to Applications. Advanced materials 23, 1154-1161 (2011).

3. Ko, F. et al. Electrospinning of continuous carbon nanotube - filled nanofiber yarns. Advanced Materials 15, 1161-1165 (2003).

4. Mylvaganam, K. \& Zhang, L.C. Ballistic resistance capacity of carbon nanotubes. Nanotechnology 18, 475701 (2007).

5. Pugno, N. The role of defects in the design of space elevator cable: From nanotube to megatube. Acta materialia 55, 5269-5279 (2007).

6. Jakubinek, M.B. et al. Thermal and electrical conductivity of array-spun multi-walled carbon nanotube yarns. Carbon 50, 244-248 (2012).

7. Behabtu, N., Green, M.J. \& Pasquali, M. Carbon nanotube-based neat fibers. Nano Today 3, 24-34 (2008).

8. Lima, M.r. et al. Electrically, chemically, and photonically powered torsional and tensile actuation of hybrid carbon nanotube yarn muscles. Science 338, 928-32 (2012).

9. Foroughi, J. et al. Torsional Carbon Nanotube Artificial Muscles. Science 334, 494497 (2011).

10. Galvan Garcia, P. et al. Robust cell migration and neuronal growth on pristine carbon nanotube sheets and yarns. Journal of biomaterials science. Polymer edition 18, 124561 (2007).

11. Lekawa Raus, A., Kurzepa, L., Peng, X. \& Koziol, K. Towards the development of carbon nanotube based wires. Carbon 68, 597-609 (2014).

12. Abbas, S. et al. Microwave Characterization of Carbon Nanotube Yarns For UWB Medical Wireless Body Area Networks. IEEE Transactions on Microwave Theory and Techniques 61, 3625-3631 (2013).

13. Schmidt, A., Wang, X., Zhu, Y. \& Sombers, L. Carbon Nanotube Yarn Electrodes for Enhanced Detection of Neurotransmitter Dynamics in Live Brain Tissue. ACS nano 7, 7864-7873 (2013).

14. Roberts, G.S. \& Singjai, P. Joining carbon nanotubes. Nanoscale 3, 4503-14 (2011).

15. Schauerman, C.M. et al. Ultrasonic Welding of Bulk Carbon Nanotube Conductors. Advanced Engineering Materials (2014).

16. $\quad \mathrm{Wu}, \mathrm{W}$. et al. Vacuum brazing of carbon nanotube bundles. Materials letters 62, 44864488 (2008).

17. Burda, M., Lekawa-Raus, A., Gruszczyk, A. \& Koziol, K.K. Soldering of Carbon Materials Using Transition Metal Rich Alloys. ACS nano 9, 8099-8107 (2015).

18. Cortizo, M.C. \& De Mele, M.F.L. Cytotoxicity of copper ions released from metal. Biological trace element research 102, 129-141 (2004).

19. Ganguly, B.B., Talukdar, G. \& Sharma, A. Cytotoxicity of tin on human peripheral lymphocytes in vitro. Mutation Research Letters 282, 61-67 (1992).

20. Lichter, S.G. et al. Hermetic diamond capsules for biomedical implants enabled by gold active braze alloys. Biomaterials 53, 464-474 (2015).

21. Ganesan, K. et al. An all-diamond, hermetic electrical feedthrough array for a retinal prosthesis. Biomaterials 35, 908-915 (2014). 
22. Sung, J. \& Sung, M. The brazing of diamond. International journal of refractory \& hard metals 27, 382-393 (2009).

23. Zhu, W.J. et al. Modeling and simulation of the TiC reaction layer growth during active brazing of diamond using DICTRA. Computational materials science 78, 74-82 (2013).

24. Artini, C., Muolo, M.L. \& Passerone, A. Diamond-metal interfaces in cutting tools: a review. Journal of materials science 47, 3252-3264 (2012).

25. Singh, M., Asthana, R., Shpargel, T.P. \& Morscher, G.N. Active metal brazing and characterization of brazed joints in titanium to carbon-carbon composites. Materials science \& engineering. A, Structural materials: properties, microstructure and processing 412, 123-128 (2005).

26. Vanhoestenberghe, A., Donaldson, N., Lovell, N. \& Suaning, G. in Conference Paper, IFESS (2008).

27. Bokhonov, B.B. et al. Towards a better understanding of nickel/diamond interactions: the interface formation at low temperatures. RSC Advances 5, 51799-51806 (2015).

28. Wörner, E., Wild, C., Müller-Sebert, W., Locher, R. \& Koidl, P. Thermal conductivity of CVD diamond films: high-precision, temperature-resolved measurements. Diamond and related materials 5, 688-692 (1996).

29. Jayasinghe, C., Amstutz, T., Schulz, M. \& Shanov, V. Improved Processing of Carbon Nanotube Yarn. Journal of Nanomaterials 2013, 1-7 (2013).

30. Pierlot, A., Woodhead, A. \& Church, J. Thermal annealing effects on multi-walled carbon nanotube yarns probed by Raman spectroscopy. Spectrochimica acta. Part A, Molecular and biomolecular spectroscopy 117, 598-603 (2014).

31. Melanitis, N., Galiotis, C. \& Tetlow, P.L. Characterization of PAN-based carbon fibres with laser Raman spectroscopy. Journal of materials science 31, 851-860 (1996).

32. Frage, N., Froumin, N. \& Dariel, M. Wetting of TiC by non-reactive liquid metals. Acta Materialia 50, 237-245 (2002).

33. Frage, N., Froumin, N. \& Dariel, M.P. Wetting of TiC by non-reactive liquid metals. Acta materialia 50, 237-245 (2002).

34. Dezellus, O. \& Eustathopoulos, N. Fundamental issues of reactive wetting by liquid metals. Journal of Materials Science 45, 4256-4264 (2010).

35. Apollo, N.V. et al. Soft, Flexible Freestanding Neural Stimulation and Recording Electrodes Fabricated from Reduced Graphene Oxide. Advanced Functional Materials (2015).

36. Abràmoff, M.D., Magalhães, P.J. \& Ram, S.J. Image processing with ImageJ. Biophotonics international 11, 36-43 (2004).

37. Stalder, A.F. et al. Low-bond axisymmetric drop shape analysis for surface tension and contact angle measurements of sessile drops. Colloids and Surfaces A:

Physicochemical and Engineering Aspects 364, 72-81 (2010).

38. Zhang, M., Atkinson, K.R. \& Baughman, R.H. Multifunctional carbon nanotube yarns by downsizing an ancient technology. Science 306, 1358-1361 (2004).

39. Jalili, R. et al. Scalable One-Step Wet-Spinning of Graphene Fibers and Yarns from Liquid Crystalline Dispersions of Graphene Oxide: Towards Multifunctional Textiles. Advanced functional materials 23, 5345-5354 (2013). 